\title{
Nanostructures in lightly doped silicon carbide crystals with polytypic defects
}

\author{
S.I. Vlaskina ${ }^{1,2}$, G.N. Mishinova ${ }^{3}$, L.V. Vlaskin ${ }^{4}$, V.E. Rodionov ${ }^{2}$, G.S. Svechnikov ${ }^{2}$ \\ ${ }^{1}$ Yeoju Institute of Technology (Yeoju University), \\ 338 Sejong-ro, Yeoju-eup, Yeoju-gun, Gyeonggi-do, 469-705 Korea \\ ${ }^{2} V$. Lashkaryov Institute of Semiconductor Physics, National Academy of Science of Ukraine, \\ 41, prospect Nauky,03028 Kyiv, Ukraine; e-mail: businkaa@mail.ru \\ ${ }^{3}$ Taras Shevchenko Kyiv National University, 64, Volodymyrs'ka str., 01033 Kyiv, Ukraine \\ ${ }^{4}$ Sensartech, 2540 Lobelia Dr., Oxnard, 93036 California, USA
}

\begin{abstract}
In this work, photoluminescence spectra of lightly doped SiC crystals with ingrown original defects are reported. Undoped $\mathrm{SiC}$ single crystals with the impurity concentration of $N_{D}-N_{A} \sim(2 \ldots 8) \cdot 10^{16} \mathrm{~cm}^{-3}, N_{A} \sim(2 \ldots 8) \cdot 10^{17} \mathrm{~cm}^{-3}$, and $N_{D}-N_{A} \sim$ (1..5) $\cdot 10^{17} \mathrm{~cm}^{-3}, N_{D} \leq 1 \cdot 10^{18} \mathrm{~cm}^{-3}$ were investigated. The analysis of absorption, excitation and low temperature photoluminescence spectra suggests formation of a new micro-phase during the growth process and appearance of the deep-level (DL) spectra. The complex spectra of the crystals can be decomposed into the so-called $\mathrm{DL}_{i}(i=1,2$, $3,4)$ spectra. The appearance of the $\mathrm{DL}_{i}$ spectrum is associated with formation of new nano-phases. Data of photoluminescence, excitation and absorption spectra show the uniformity of different $\mathrm{DL}_{i}$ spectra. Structurally, the general complexity of the $\mathrm{DL}_{i}$ spectra correlated with the degree of disorder of the crystal and was connected with onedimensional disorder, the same as in the case of the stacking fault $\left(\mathrm{SF}_{i}\right)$ spectra. The $\mathrm{DL}_{i}$ spectra differ from $\mathrm{SF}_{i}$ spectra and have other principles of construction and behavior. The $\mathrm{DL}_{i}$ spectra are placed on a broad donor-acceptor pairs emission band in crystals with higher concentrations of non-compensated impurities. The excitation spectra for the $\mathrm{DL}_{i}$ and $\mathrm{SF}_{i}$ spectra coincide and indicate formation of nanostructures $14 \mathrm{H}_{1}\langle 4334\rangle$, $10 \mathrm{H}_{2}\langle 55\rangle, 14 \mathrm{H}_{2}\langle 77\rangle, 8 \mathrm{H}\langle 44\rangle$.
\end{abstract}

Keywords: silicon carbide, polytype, stacking fault, photoluminescence spectra, nanostructure.

Manuscript received 15.01.14; revised version received 29.04.14; accepted for publication 12.06.14; published online 30.06.14.

\section{Introduction}

Silicon carbide $(\mathrm{SiC})$ is material widely used in several industrial applications such as high power electronics, light emitting diodes, as well as in researches such as photo-voltaic and quantum technologies. Silicon carbide exists as several different polytypes according to the stacking sequence of $\mathrm{SiC}$ atomic double layers in the crystal lattice. Great advances were reported in growing a single crystalline polytype by controlled sublimation and chemical vapor deposition [1-3]. Polytypic transformation in $\mathrm{SiC}$ was investigated by many authors [4-19].

The phase transformation in highly doped by nitrogen $6 \mathrm{H}-\mathrm{SiC}$ crystal and the mechanism of solidphase transition through formation of multilayer polytypes has been studied $[5,14]$. Syntax coalescence becomes a very important characteristic of $\alpha$-polytype $\mathrm{SiC}$ crystals - the intergrowth of two or more polytypes 
when not only the major axis $c$ but also the axis $a$ coincide in the hexagonal structures. It happens because of the "layer-by-layer" crystal growth and the existence of different types of errors in the layers sequence (SF) along the axis $c$. The layers with in-grown one dimensional disorder in the original $\alpha$-SiC crystals are called D-layers. D-layers have a clear crisp coalescence boundary with the neighboring blocks of the structurally perfect $6 \mathrm{H}-\mathrm{SiC}$ along the (0001) plane.

However, single crystals with multiple nucleations also exist. The interaction of the growth fronts produces incoherent boundaries (the so-called "mosaic"). In this case, all kinds of in-grown dislocations and properties of "border inconsistencies" play a crucial role. To stabilize the appropriate polytype structure, it is essential to consider the influence of different factors: thermodynamic, kinetic, impurity, and dislocation nucleation mechanisms. Any fluctuation of the stabilization parameters can lead to errors in structure formation. It also contributes to a very small stacking fault energy in silicon carbide $\left(2-9 \mathrm{~mJ} / \mathrm{m}^{2}\right)$.

Photoluminescence (PL) is one of the most widely used experimental techniques for the physical characterization of semiconductors. The complex research of the low temperature photoluminescence (LTPL) spectra under different conditions of registration and at various temperatures is one among the most widely used experimental techniques for the physical characterization of the crystals $[3,6,17]$. The simultanious control of the phase state and structural imperfactions showed defect formation as a result of polytypes reorganization in the process of crystal growth.

Investigated in the paper [5] have been defects in heavily doped with nitrogen single crystals by using photoluminescence. However, correspondence between the PL spectra and mechanism of phase transformation has not been investigated. The nature of the PL spectra depends on the concentration of impurities. Spectroscopy of defects in pure perfect crystal $\mathrm{SiC}$ has already been described in the works of Refs. [6, 16, 20-22].

In this report, LTPL studies of lightly doped $\alpha$-SiC single crystals have been presented. This work is the continuation of the spectroscopic diagnosis of defects created by the unstable growth conditions in lightly doped $\alpha-\mathrm{SiC}$ single crystals.

\section{Experimental}

The method of optical spectroscopy LTFL was used in this work, as it is very sensitive to structural changes.

The group of single crystalline $\alpha$-SiC crystals grown by using Lely's (Tairov's) method [3] was selected for the research. Undoped SiC single crystals with the impurity concentration of $N_{D}-N_{A} \sim$ $(2 \ldots 8) \cdot 10^{16} \mathrm{~cm}^{-3}, N_{A} \sim(2 \ldots 8) \cdot 10^{17} \mathrm{~cm}^{-3}$, and $N_{D}-N_{A} \sim$ (1...5) $10^{17} \mathrm{~cm}^{-3}, N_{D} \leq 1 \cdot 10^{18} \mathrm{~cm}^{-3}$ were investigated. In perfect $6 \mathrm{H}-\mathrm{SiC}$ crystals, the donor impurity $(\mathrm{N})$ and the acceptor impurity (Al) in low-temperature recombination show well-known blue radiation of donor-acceptor pairs (DAP). This type of recombination usually dominates [3].

We selected samples with LTPL $(77 \mathrm{~K})$ in the turquoise green spectrum area. We will call these samples deep level (DL) samples. The turquoise green luminescence was observed within the whole crystal volume as well as only in some parts of the crystal. For example, in the block from one side of the (0001) $\mathrm{SiC}$ crystal, the DL luminescence was observed while the other part of the crystal produced the DAP $(6 \mathrm{H}-\mathrm{SiC})$ luminescence. These blocks were separated from the rest of the crystal by using layer-by-layer polishing and were selected for further structural analysis.

The X-ray (Laue's method) and electron diffraction methods were used to detect symmetrical and layering parameters of the crystals. Moreover, in some cases, to analyze structural peculiarities of the allocated blocks, the transmission electron microscopy technique (TEM) $(\times 15000)$ was used. The metallographic method was used for evaluating the crystal face surface in the starting growth state.

LTPL spectra were registered by the ДФС-12 spectrograph with the photodetector (ФЭУ-79). In photoluminescence (PL) experiments, a nitrogen ЛГИ-21, $\lambda=337 \mathrm{~nm}(3.68 \mathrm{eV})$ or helium-cadmium ЛГ-70, $\lambda=$ $441.6 \mathrm{~nm}(2.807 \mathrm{eV})$ laser, respectively, were used. Also, a mercury ultrahigh pressure lamp СВДШ-1000 with УФ-2 filter and xenon lamp ДКСШ-1000 were used.

The PL spectra were measured using the samples contained in a liquid helium or nitrogen cryostat, which provided temperatures ranging from 1.5 to $330 \mathrm{~K}$.

\section{Results and discussions}

$\mathrm{SF}$ related luminescence dominates in pure $\mathrm{SiC}$ crystals [22]. After full extinction of the $\mathrm{SF}_{i}$ luminescence described in [23] at the temperature of $35-40 \mathrm{~K}$, a weak (in its intensity) and wide background band of radiation remained. This luminescence was more or less clearly pronounced and had a rich structure depending on the features and degree of the one-dimensional disorder of the samples.

The spectra of this type $\left(\mathrm{DL}_{i}\right)$ differ from $\mathrm{SF}_{i}$ spectra and have other principles of construction and behavior. The $\mathrm{DL}_{i}$ spectra are localized on the background of the broad DAP spectral band of crystals with higher concentrations of non-compensated impurities. Originally, these spectra were found and investigated in defective crystals $\left(1_{\mathrm{DL}}, 2_{\mathrm{DL}}\right.$ samples in Fig. 1) with a higher concentration of the main impurity. In these samples, the DL spectrum was much more intensive, whereas the SF spectrum (which was also present) even at $4.2 \mathrm{~K}$ was poorly pronounced, or practically was not fixed.

The dependence of the intensity of the $\mathrm{DL}_{i}$ spectra on the impurity concentration is shown in Fig. 1. 
Fig. 2 shows absorption spectra of $\mathrm{DL}_{i}$ samples. Samples with $\mathrm{DL}_{1}$ spectra have a more or less pronounced sharp absorption edge (typical for $6 \mathrm{H}-\mathrm{SiC}$ crystals) that transforms into a long-wave smooth tail, testifying to the existence of absorption in the transparency region of this phase. There are no evidence that this absorption is related with the impurity concentration $\left(N_{D}-N_{A} \sim(3 \ldots 8) \cdot 10^{16} \mathrm{~cm}^{-3}\right)$ in the samples.

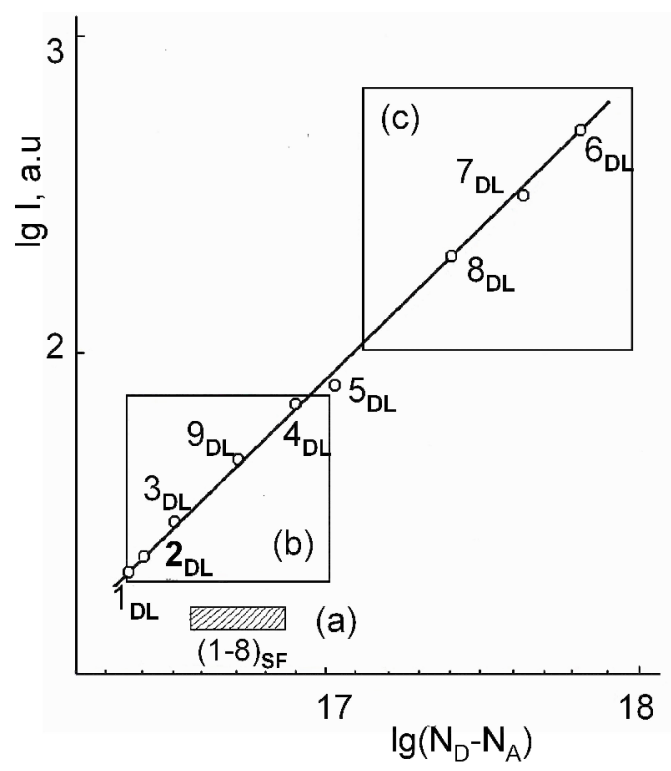

Fig. 1. Intensity of the $\mathrm{DL}_{i}$ spectra $(i=1)(T=77 \mathrm{~K})$ at $2.4 \ldots 2.5 \mathrm{~V}$ vs. the impurity concentration for different samples (of the $\mathrm{SF}_{i}$ and $\mathrm{DL}_{i}$ series). Shaded area - the $\mathrm{SF}_{i}$ spectra from the ref. [22]. (a) Most pure samples with only SF spectra, $N_{D}-N_{A} \sim(4 \ldots 9) \cdot 10^{16} \mathrm{~cm}^{-3},(1 \ldots .5) \cdot 10^{16} \mathrm{~cm}^{-3}, N_{D}<1 \cdot 10^{17} \mathrm{~cm}^{-3}$, $N_{A} \sim(1 \ldots 3) \cdot 10^{16} \mathrm{~cm}^{-3}$ from the ref. [22]. (b) Pure samples with the $\mathrm{SF}_{i}$ and $\mathrm{DL}_{i}$ series, $N_{D}-N_{A} \sim(2 \ldots 8) \cdot 10^{16} \mathrm{~cm}^{-3}, N_{A} \sim$ $(2 \ldots 8) \cdot 10^{17} \mathrm{~cm}^{-3}$. (c) Samples with only $\mathrm{DL}_{i}$ spectra, $N_{D}-$ $N_{A} \sim(2 \ldots 7) \cdot 10^{17} \mathrm{~cm}^{-3}, N_{D}<1 \cdot 10^{18} \mathrm{~cm}^{-3}$.

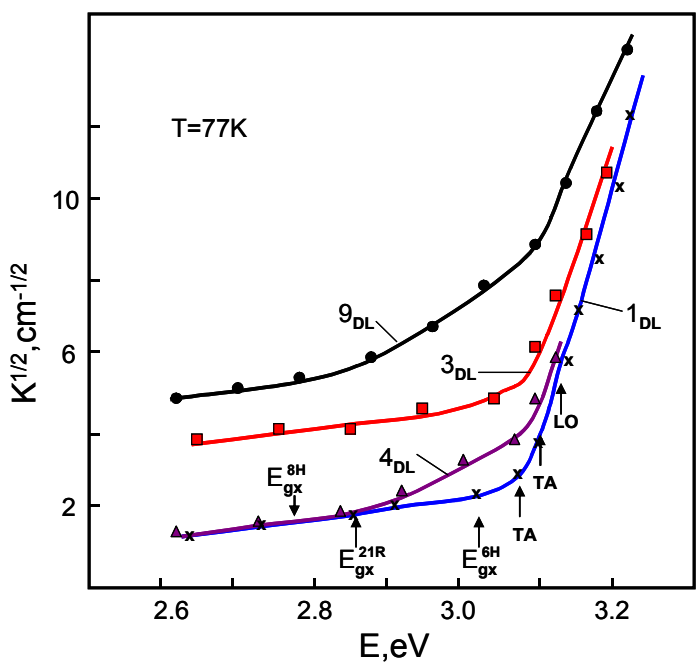

Fig. 2. Absorption spectra of DL samples.
However, the shift to the longwave area and absorption coefficient fully correlate with the degree and character of structural defects in the samples. The absorption area is located far away in the longwave side in relation to the exciton absorption edge $\left(E_{g x}\right)$ of $6 \mathrm{H}-\mathrm{SiC}$. It rather strongly suggests formation of structures with a higher percentage of cubic $\alpha$-SiC than $6 \mathrm{H}-\mathrm{SiC}$. The peculiarities of the absorption spectra are also in good agreement with those for the $\mathrm{DL}_{i}$ excitation spectra. The absorption at the energy $E<E_{g x} 6 \mathrm{H}-\mathrm{SiC}$ can be attributed to the absorption of the new matrix of micro- or nano-phases, as noted in the analysis/study of the $\mathrm{SF}_{i}$ spectra [22].

The PL spectra $(77 \mathrm{~K})$ and corresponding excitation low temperature photoluminescence spectra (ELTPLS) of these DL samples are shown in Fig. 3.

There is correlation between the energy shift of longwave edges of the excitation low temperature PL spectra and the corresponding $\mathrm{DL}_{i}$ spectra. Consequently, it is possible to eliminate completely shorter wavelength $\mathrm{DL}_{i}$ spectra from the total complex panorama of the $\mathrm{DL}_{i}$ spectra (for example as it is shown for $9_{\mathrm{DL}}$ sample) by choosing suitable photon energy of the exciting radiation. It was done using monochromatic light and various lasers.

Structurally, the total complexity of the $\mathrm{DL}_{i}$ spectra correlated with the degree of disorder of the crystal and was related with peculiarities of defect characteristics performance (as in the case of the $\mathrm{SF}_{i}$ spectra [22]) and, above all, with such its manifestations as onedimensional disordering. The complex spectrum of the crystals can be decomposed into the so-called $\mathrm{DL}_{i}(i=1$, 2, 3, 4) spectra.

As it is shown in Fig. 3, the PL spectra of the $1_{\mathrm{DL}}$ and $8_{\mathrm{DL}}$ samples are represented mainly by only one $\mathrm{DL}_{1}$ and $\mathrm{DL}_{2}$ spectra, respectively, while the sample $9_{\mathrm{DL}}$ includes more than one $\mathrm{DL}_{3}, \mathrm{DL}_{4}$ spectra, which corresponds to an even higher degree of the structural disordering of the crystals.

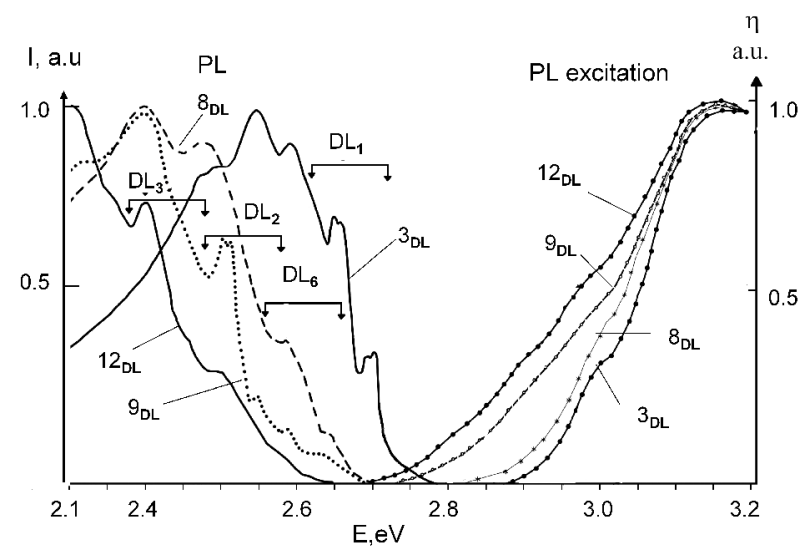

Fig. 3. $\mathrm{DL}_{i}$ spectra (I) and corresponding photoluminescence excitation spectra $(\eta)$ at $T=77 \mathrm{~K}$. The longwave edge of the photoluminescence excitation spectra for the $\mathrm{DL}_{i}$ and $\mathrm{SF}_{i}$ types is the same $\left(i=1 \rightarrow E_{g x}(21 \mathrm{R}), i=2 \rightarrow E_{g x}\left(10 \mathrm{H}_{2}\right), i=3 \rightarrow E_{g x}\right.$ $\left(14 \mathrm{H}_{2}\right), \ldots, i=6 \rightarrow E_{g x}(8 \mathrm{H})$. 


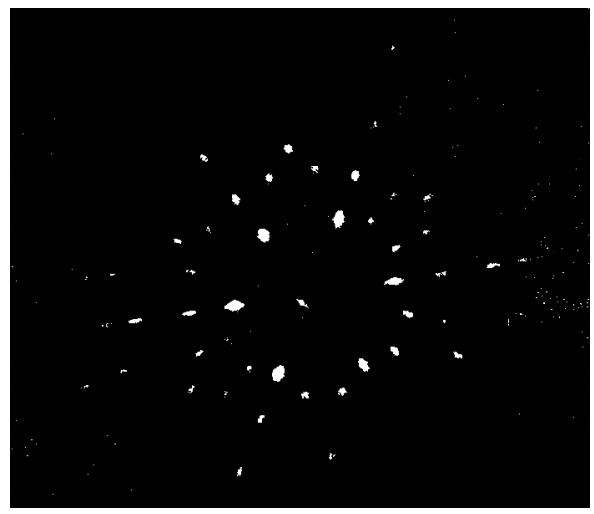

a)

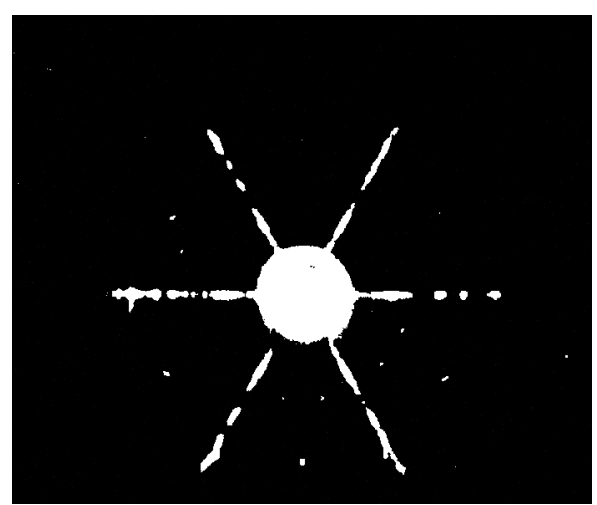

b)

Photo 1. Laue pattern of SiC crystals with DL luminescence spectra: a) sample $4_{\mathrm{DL}}$, b) sample $9_{\mathrm{DL}}$

Comparison of Laue patterns (Photo 1) shows that the crystal 9 $9_{\mathrm{DL}}$ looks like "morphological freak". Following changes in DL-luminescence in this crystal with layer-by-layer grinding of the (0001) face (along the axis $c$ ), it was possible to trace transformation of the structure during the growth process. The spectrum from the base surface of one of the faces of the crystal in the original state of the sample $9_{\mathrm{DL}}$ is dominated by $\mathrm{DL}_{3}$ and weaker $\mathrm{DL}_{2}$ and $\mathrm{DL}_{1}$ spectra, then with the grinding of the defect layers, $\mathrm{DL}_{2}$ luminescence was mainly observed, and from the other natural face $\mathrm{DL}_{1}$ luminescence took place. Thus, the luminescence within one sample reflects distribution of the corresponding luminescence centers during the growth process and indicates the independence of each of the $\mathrm{DL}_{i}$ spectra.

Comparison of the longwave edge of these samples with the longwave edges of the $\mathrm{SF}_{i}$ type samples (pure crystals) (Fig. 4) [21, 22] shows evidence that the ELTPLS for $\mathrm{DL}_{i}$ type spectra and ELTPLS for $\mathrm{SF}_{i}$ type coincide:

$$
\begin{aligned}
& i=1 \rightarrow E_{g x}\left(14 \mathrm{H}_{1}\right)\langle 4334\rangle, \\
& i=2 \rightarrow E_{g x}\left(10 \mathrm{H}_{2}\right)\langle 55\rangle, \\
& i=3 \rightarrow E_{g x}\left(14 \mathrm{H}_{2}\right)\langle 77\rangle, \\
& i=4 \rightarrow E_{g x}(\mathrm{~N} / \mathrm{A}), \\
& i=5 \rightarrow E_{g x}(33 \mathrm{R})\left\langle(3332)_{3}\right\rangle, \\
& i=6 \rightarrow E_{g x}(8 \mathrm{H})\langle 44\rangle .
\end{aligned}
$$

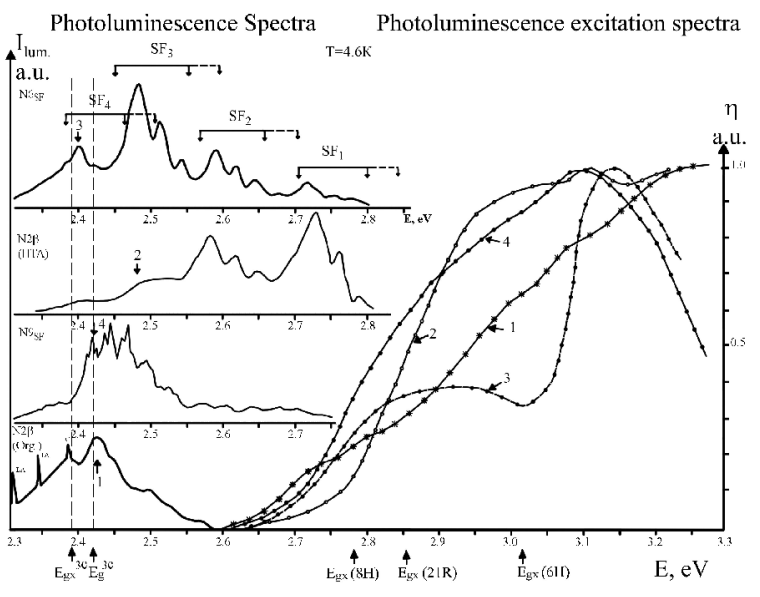

Fig. 4. $\mathrm{SF}_{i}$ spectra and corresponding photoluminescence excitation spectra (ELTFLS) at $T=4.6 \mathrm{~K}$. The longwave edge of ELTFLS for $\mathrm{DL}_{i}$ and $\mathrm{SF}_{i}$ types is the same $\left(i=1 \rightarrow E_{g x}\right.$ $\left(14 \mathrm{H}_{1}\right), i=2 \rightarrow E_{g x}\left(10 \mathrm{H}_{2}\right), i=3 \rightarrow E_{g x}\left(14 \mathrm{H}_{2}\right)$. Arrows $(\downarrow, \uparrow)$ indicate the areas of the PL spectra for which the excitation spectra were recorded.

Thus, the analysis of the excitation spectra of the $\mathrm{DL}_{i}$ luminescence leads to the conclusion that the spectra are in qualitative agreement with the excitation spectrum of ELTPLS luminescence for the appropriate type $\mathrm{SF}_{i}$ $\left(\right.$ ELTPLS $\mathrm{DL}_{1} \rightarrow$ ELTPLS $\mathrm{SF}_{1}$, ELTPLS $\mathrm{DL}_{2} \rightarrow$ ELTPLS $\mathrm{SF}_{2}$ ). Consequently, DL luminescence is related to formation of a new micro-phase during the growth, and DL spectra are also indicators of the corresponding motif forming [23] the main phase adjustment.

\section{Conclusion}

In this work, photoluminescence spectra of lightly doped $\mathrm{SiC}$ crystals with in-grown original defects are reported. Undoped $\mathrm{SiC}$ single crystals with the impurity concentration of $N_{D}-N_{A} \sim(2 \ldots 8) \cdot 10^{16} \mathrm{~cm}^{-3}, \quad N_{A} \sim$ $(2 \ldots 8) \cdot 10^{17} \mathrm{~cm}^{-3}, \quad$ and $N_{D}-N_{A} \sim(1 \ldots 5) \cdot 10^{17} \mathrm{~cm}^{-3}$, $N_{D} \leq 1 \cdot 10^{18} \mathrm{~cm}^{-3}$ were investigated. The analysis of absorption, excitation and low temperature photoluminescence spectra of lightly doped SiC crystals with in-grown original defects suggests formation of a new micro-phase during the growth process and appearance of the deep-level (DL) spectra. The complex spectra of the crystals can be decomposed into the socalled $\mathrm{DL}_{i}(i=1,2,3,4)$ spectra. The appearance of the $\mathrm{DL}_{i}$ spectrum is associated with formation of new nanophases. Data of photoluminescence, excitation and absorption spectra show the uniformity of different $\mathrm{DL}_{i}$ spectra. Structurally, the general complexity of the $\mathrm{DL}_{i}$ spectra correlated with the degree of disorder of the crystal and was connected with one-dimensional disorder, the same as in the case of the stacking fault $\left(\mathrm{SF}_{i}\right)$ spectra. The $\mathrm{DL}_{i}$ spectra differ from $\mathrm{SF}_{i}$ spectra and have other principles of construction and behavior. 
The $\mathrm{DL}_{i}$ spectra are placed on a broad donor-acceptor pairs emission band in crystals with higher concentrations of non-compensated impurities. The excitation spectra for the $\mathrm{DL}_{i}$ and $\mathrm{SF}_{i}$ spectra coincide and indicate formation of nanostructures $14 \mathrm{H}_{1}\langle 4334\rangle$, $10 \mathrm{H}_{2}\langle 55\rangle, 14 \mathrm{H}_{2}\langle 77\rangle, 8 \mathrm{H}\langle 44\rangle$.

\section{References}

1. http://www.imicronews.com/upload/Rapports/Yole Patentanalysis_of_SiC_single_crystal_wafer_and_e piwafer_manufacturer_July_sample_2012.pdf

2. Fei Yan, dissertation "Low Temperature Study on Defect Centers in Silicon Carbide", University of Pittsburgh, 2009 (Dissertation LTPL-Choyke Pittsburg 2009 pdf).

3. W.J. Choyke, H. Matsunami, Silicon Carbide: Recent Major Advances books.google.co.kr/ books?isbn $=3540404589$ Gerhard Pensl -2004 .

4. P. Pirouz, M. Zhang, H. McD. Hobgood, M. Lancin, J. Douin, and B. Pichaud, Nitrogen doping and multiplicity of stacking faults in $\mathrm{SiC} / /$ Phil. Mag. A, 86 (29-31), p. $4685-4697$ (2006).

5. S.I. Vlaskina, D.H. Shin, $6 \mathrm{H}$ to $3 \mathrm{C}$ polytype transformation in silicon carbide // Jpn. J. Appl. Phys. 38, p. 27-29 (1999).

6. I.S. Gorban and G.N. Mishinova, Basics of luminescent diagnostics of the dislocation structure of SiC crystals // Proc. SPIE, 3359, p. 187 (1998).

7. A. Galeckas, H. K. Nielsen, J. Linnros, A. Hallén, B.G. Svensson, and P. Pirouz, Investigation of stacking fault formation in hydrogen bombarded 4H-SiC // Mater. Sci. Forum, 483-485, p. 327-330 (2005).

8. S.I. Maximenko, T. Sudarshan, and P. Pirouz, Investigation of the electrical activity of partial dislocations in SiC p-i-n diodes // Appl. Phys. Lett. 87(3), 033503-0 - 033503-3 (2005).

9. A. Galeckas, J. Linnros, P. Pirouz, Recombination induced stacking faults: Evidence for a general mechanism in hexagonal SiC // Phys. Rev. Lett. 96(2), 025502-1 - 025502-4 (2006).

10. S.I. Maximenko, P. Pirouz, and T.S. Sudarshan, Open core dislocations and surface energy of SiC // Mater. Res. Forum, 527-529, p. 439-442 (2006).

11. H. Idrissi, B. Pichaud, G. Regula, and M. Lancin, $30^{\circ} \mathrm{Si}$ partial dislocation mobility in nitrogendoped 4H-SiC // J. Appl. Phys. 101, 113533 (2007).

12. G.R. Fisher, P. Barnes, Towards a unified view of polytypism in silicon carbide // Phil. Mag., Part B, 1463-6417, 61(2), p. 217-236 (1990).

13. Sh. Sugiyama, M. Togaya, Phase relationship between 3C- and 6H-silicon carbide at high pressure and high temperature // J. Amer. Cer. Soc. 84(12), p. 3013-3016 (2001).
14. S.I. Vlaskina, Mechanism of 6H-3C transformation in $\mathrm{SiC} / /$ Semiconductor Physics, Quantum Electronics and Optoelectronics, 5(2), p. 252-255 (2002).

15. M. Durandurdu, An initio simulations of the structural phase transformation of $2 \mathrm{H}-\mathrm{SiC}$ at high pressure // Phys. Rev. B, 75, 235204 (2007).

16. S.W. Lee, S.I. Vlaskina, V.I. Vlaskin, I.V. Zaharchenko, V.A. Gubanov, G.N. Mishinova, G.S. Svechnikov, V.E. Rodionov, S.A. Podlasov, Silicon carbide defects and luminescence centers in current heated 6H-SiC // Semiconductor Physics, Quantum Electronics and Optoelectronics, 13(1), p. 24-29 (2010).

17. C. Raffy, Furthmüller and Beshtedt, Properties of interface between cubic and hexagonal polytypes in silicon carbide // J. Phys.: Condens. Matter, 14, p. 12725-12731 (2002).

16. F. Shimojo, I. Ebbsjö, R.K. Kalia, A. Nakano, J.P. Rino, and P. Vashishta, Molecular dynamics simulation of structural transformation in silicon carbide under pressure // Phys. Rev. Lett. 84, p. 3338-3341 (2000).

17. S. Juillaguet, T. Robert, J. Camassel, Optical investigation of stacking faults in $4 \mathrm{H}-\mathrm{SiC}$ epitaxial layers: Comparison of $3 \mathrm{C}$ and $8 \mathrm{H}$ polytypes // Mater. Sci. and Eng. B, 165, p. 5-8 (2009).

18. S. Shinozaki, K.R. Kisman, Aspects of "one dimensional disorder" in silicon carbide // Acta Metallurgica, 26, p. 769-776 (1978).

19. L.U. Ogbuji, T.E. Mitchell, A.H. Heuer, The $\beta \rightarrow \alpha$ transformation in polycrystalline $\mathrm{SiC}$ : The thickening of $\alpha$ plates // J. Amer. Ceram. Soc. 64(2), p. 91-99 (1981).

20. S.I. Vlaskina, G.N. Mishinova, V.I. Vlaskin, V.E. Rodionov, G.S. Svechnikov, 6H-3C transformation in heated cubic silicon carbide 3C-SiC // Semiconductor Physics, Quantum Electronics and Optoelectronics, 14(4), p. $432-437$ (2013).

21. S.I. Vlaskina, G.N. Mishinova, V.I. Vlaskin, G.S. Svechnikov, V.E. Rodionov, S.W. Lee, Silicon carbide phase transition in as-grown 3C-6H polytypes junction // Semiconductor Physics, Quantum Electronics and Optoelectronics, 16(2), p. 132-136 (2013).

22. S.I. Vlaskina, G.N. Mishinova, V.I. Vlaskin, V.E. Rodionov, G.S. Svechnikov, 8H-, 10H-, 14H-SiC formation in $6 \mathrm{H}-3 \mathrm{C}$ silicon carbide phase transitions // Semiconductor Physics, Quantum Electronics and Optoelectronics, 16(3), p. 272-278 (2013).

23. Kozuaki Kobayashi, Shojiro Komatsu. Firstprinciple study of $8 \mathrm{H}-, 10 \mathrm{H}-, 12 \mathrm{H}-$, ND $18 \mathrm{H}-\mathrm{SiC}$ polytypes // J. Phys. Soc. Jpn. Appl. 024714 (2012). 